MNHMT2009-18032

\title{
EFFECT OF DIAMOND NANOLUBRICANT ON R134A POOL BOILING HEAT TRANSFER
}

\author{
Mark A. Kedzierski \\ National Institute of Standards and Technology \\ Gaithersburg, MD 20899 \\ Email: Mark.Kedzierski@NIST.gov
}

\section{ABSTRACT}

This paper quantifies the influence of diamond nanoparticles on the pool boiling performance of $\mathrm{R} 134 \mathrm{a} /$ polyolester mixtures on a roughened, horizontal, flat surface. Nanofluids are liquids that contain dispersed nanosize particles. A lubricant based nanofluid (nanolubricant) was made by suspending $10 \mathrm{~nm}$ diameter diamond particles in a synthetic ester to roughly a $2.6 \%$ volume fraction. For the $0.5 \%$ nanolubricant mass fraction, the nanoparticles caused a heat transfer enhancement relative to the heat transfer of pure R134a/polyolester (99.5/0.5) as large as $129 \%$ for the best performing tests. A similar enhancement was observed for the R134a/nanolubricant (99/1) mixture, which had a heat flux that was on average $91 \%$ larger than that of the R134a/polyolester (99/1) mixture. Further increase in the nanolubricant mass fraction to $2 \%$ resulted in boiling heat transfer degradation of approximately $19 \%$ for the best performing tests. It was speculated that the poor quality of the nanolubricant suspension caused the performance of the (99.5/0.5), and the (98/2) nanolubricant mixtures to decay over time to, on average, $36 \%$ and $76 \%$ of the of pure R134a/polyolester performance, respectively. Thermal conductivity and viscosity measurements and a refrigerantllubricant mixture pool-boiling model were used to suggest that increases in thermal conductivity and lubricant viscosity are mainly responsible for the heat transfer enhancement due to nanoparticles. Particle size measurements were used to suggest that particle agglomeration induced a lack of performance repeatability for the (99.5/0.5) and the (98/2) mixtures. From the results of the present study, it is speculated that if a good dispersion of nanoparticles in the lubricant is not obtained, then the agglomerated nanoparticles will not provide interaction with bubbles, which is favorable for heat transfer. Further research with nanolubricants and refrigerants are required to establish a fundamental understanding of the mechanisms that control nanofluid heat transfer.

\author{
NOMENCLATURE \\ English Symbols \\ $L_{\mathrm{y}}$ length of test surface (Fig. 2), $\mathrm{m}$ \\ $q$ " average wall heat flux, $\mathrm{W} \mathrm{m}^{-2}$ \\ $T$ temperature, $\mathrm{K}$ \\ $T_{\mathrm{w}}$ temperature at roughened surface, $\mathrm{K}$ \\ $U$ expanded uncertainty \\ $u_{\mathrm{i}} \quad$ standard uncertainty \\ Greek symbols \\ $\Delta T$ temperature difference, $\mathrm{K}$ \\ $\Delta T_{\mathrm{s}}$ wall superheat: $T_{\mathrm{w}}-T_{\mathrm{s}}, \mathrm{K}$ \\ English Subscripts \\ C diamond nanoparticles \\ p pure R134a \\ PL R134a/RL68H mixture \\ q" heat flux \\ s saturated state \\ Tw wall temperature \\ w wall, heat transfer surface
}

\section{INTRODUCTION}

Energy efficiency is a primary component for net zero energy, high performance green building-design (OSTP 2008, EPA 2008). Chillers that provide air conditioning for buildings consume nearly $13 \%$ of total building electric expenditures (EIA, 2008). Consequently, a cost-effective means for improving the efficiency of chillers would facilitate meeting green building goals. In recent years, nanofluids, i.e., liquids with dispersed nano-size particles, have been shown to be a potential means for enhancing the performance of chillers. For example, Liu et al. (2009) have demonstrated that a waterbased nanofluid in the evaporator improved the efficiency of a 
laboratory chiller by more than $5 \%$. Given that nanoparticles are not likely to be trapped by filters or increase abrasion because of their small size (Bahrami et al., 2006), they appear to be a viable option for improving chiller performance.

In addition to water-based nanofluids, lubricant-based nanofluids, i.e., nanolubricants, have been used to improve refrigerant boiling heat transfer that will, likewise, benefit refrigerant cycle performance. For example, recent studies by Kedzierski (2009a) and Bi et al. (2007) have recommended the use of nanolubricants as a means for improving efficiencies of chillers and refrigerators, respectively. In addition, Marquis and Chibante (2005) discuss the improved lubricating qualities of nanolubricants for engines. In a similar manner for engines, improved lubricity is also expected to benefit the performance of the chiller compressor by reducing power requirements.

Nanoparticle properties are crucial for determining the performance characteristics of nanolubricants. According to Bobbo et al. (2009), the way in which nanoparticle material, dimension, shape and concentration affect nanolubricant properties is complex and not well understood. Marquis and Chibante (2005) point out that nanoparticle size is more influential in determining thermal conductivity than is the shape of the nanoparticle. Kedzierski (2009a) has shown that the concentration of $\mathrm{CuO}$ nanoparticles may determine whether an enhancement or a degradation in refrigerant/lubricant boiling heat transfer is obtained. The same study also showed that the improvement in thermal conductivity was not the governing factor in determining the magnitude of the enhancement despite $\mathrm{CuO}$ having nearly two-orders-ofmagnitude greater thermal conductivity than the base lubricant. Clearly, knowledge of how the properties of nanoparticles influence the heat transfer behavior of nanolubricants must be obtained before the performance can be optimized.

In order to further investigate the influence of nanoparticle thermal conductivity on refrigerant/lubricant pool boiling, the boiling heat transfer of three R134a/nanolubricant mixtures on a roughened, horizontal, flat (plain), copper surface were measured. A commercial polyolester lubricant $(\mathrm{RL} 68 \mathrm{H})^{1}$ with a nominal kinematic viscosity of $72.3 \mu \mathrm{m}^{2} / \mathrm{s}$ at $313.15 \mathrm{~K}$ was the base lubricant that was mixed with nominally $10 \mathrm{~nm}$ diameter, dry diamond nanoparticles. Diamond nanoparticles have many commercial applications including use in polishing, tooling and die work. The RL68H/diamond mixture (nanolubricant) was made by ultrasonically mixing commercial diamond nanoparticles without a surfactant with pure RL68HA over a fivemonth period. The mixture was made such that $2.6 \%$ of the volume was diamond particles. The volume faction was chosen so that it was large enough to give a large thermal conductivity, while small enough so that the viscosity would be acceptable

\footnotetext{
${ }^{1}$ Certain commercial equipment, instruments, or materials are identified in this paper in order to specify the experimental procedure adequately. Such identification is not intended to imply recommendation or endorsement by the National Institute of Standards and Technology, nor is it intended to imply that the materials or equipment identified are necessarily the best available for the purpose.
}

for compressor applications. The particle size and dispersion was measured with a light scattering technique and a sieving technique and was found to have a wide range of agglomerated particles between approximately $10 \mathrm{~nm}$ and $250 \mu \mathrm{m}$. The RL68H/diamond $(97.4 / 2.6)^{2}$ volume fraction mixture, a.k.a. RL68H2C, was mixed with pure R134a to obtain three R134a/RL68H2C mixtures at nominally $0.5 \%, 1 \%$, and $2 \%$ RL68H2C mass fractions for the boiling tests. In addition, the boiling heat transfer of three R134a/RL68H mixtures $(0.5 \%$, 1 $\%$, and $2 \%$ mass fractions), without nanoparticles, was measured to serve as a baseline for comparison to the RL68H2C mixtures.

\section{APPARATUS}

Figure 1 shows a schematic of the apparatus that was used to measure the pool boiling data of this study. More specifically, the apparatus was used to measure the liquid saturation temperature $\left(T_{\mathrm{s}}\right)$, the average pool-boiling heat flux $\left(q^{\prime \prime}\right)$, and the wall temperature $\left(T_{\mathrm{w}}\right)$ of the test surface. The three principal components of the apparatus were the test chamber, the condenser, and the purger. The internal dimensions of the test chamber were $25.4 \mathrm{~mm} \times 257 \mathrm{~mm} \times$ $1.54 \mathrm{~m}$. The test chamber was charged with approximately 7 $\mathrm{kg}$ of refrigerant, giving a liquid height of approximately 80 $\mathrm{mm}$ above the test surface. As shown in Fig. 1, the test section was

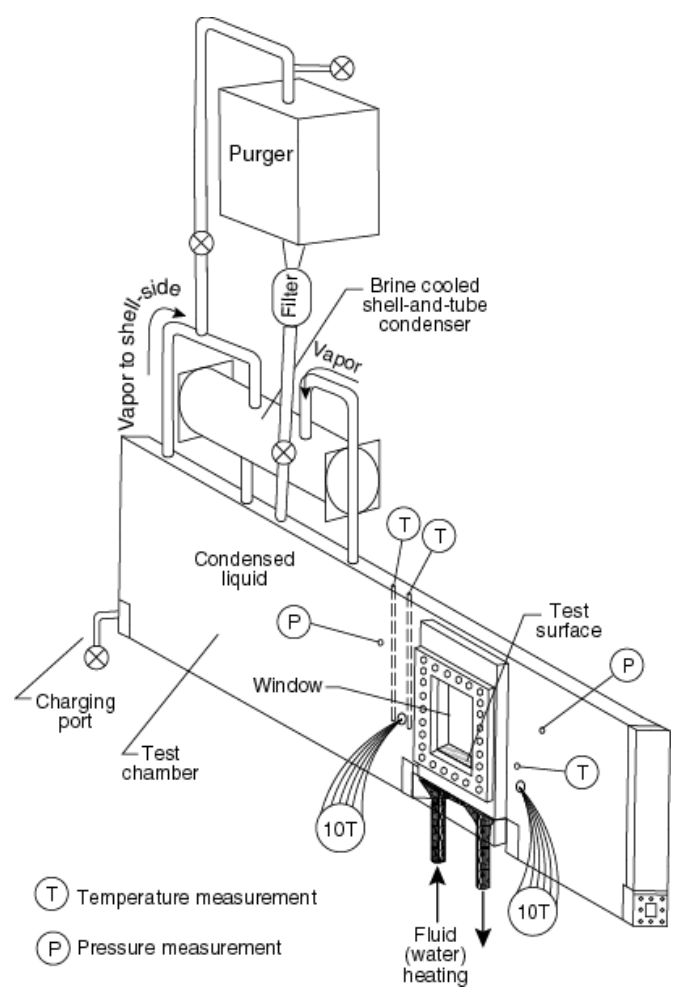

Fig. 1 Schematic of test apparatus

\footnotetext{
${ }^{2}$ The equivalent mixture is RL68H/diamond (91.3/8.7) in terms of mass.
} 
visible through two opposing, flat $150 \mathrm{~mm} \times 200 \mathrm{~mm}$ quartz windows. The bottom of the test surface was heated with high velocity $(2.5 \mathrm{~m} / \mathrm{s})$ water flow. The vapor produced by liquid boiling on the test surface was condensed by the brine-cooled, shell-and-tube condenser and returned as liquid to the pool by gravity. Further details of the test apparatus can be found in Kedzierski (2002) and Kedzierski (2001a).

\section{TEST SURFACE}

Figure 2 shows the oxygen-free high-conductivity (OFHC) copper flat test plate used in this study. The test plate was machined out of a single piece of OFHC copper by electric discharge machining (EDM). A tub grinder was used to finish the heat transfer surface of the test plate with a crosshatch pattern. Average roughness measurements were used to estimate the range of average cavity radii for the surface to be between $12 \mu \mathrm{m}$ and $35 \mu \mathrm{m}$. The relative standard uncertainty of the cavity measurements were approximately $\pm 12 \%$. Further information on the surface characterization can be found in Kedzierski (2001a).

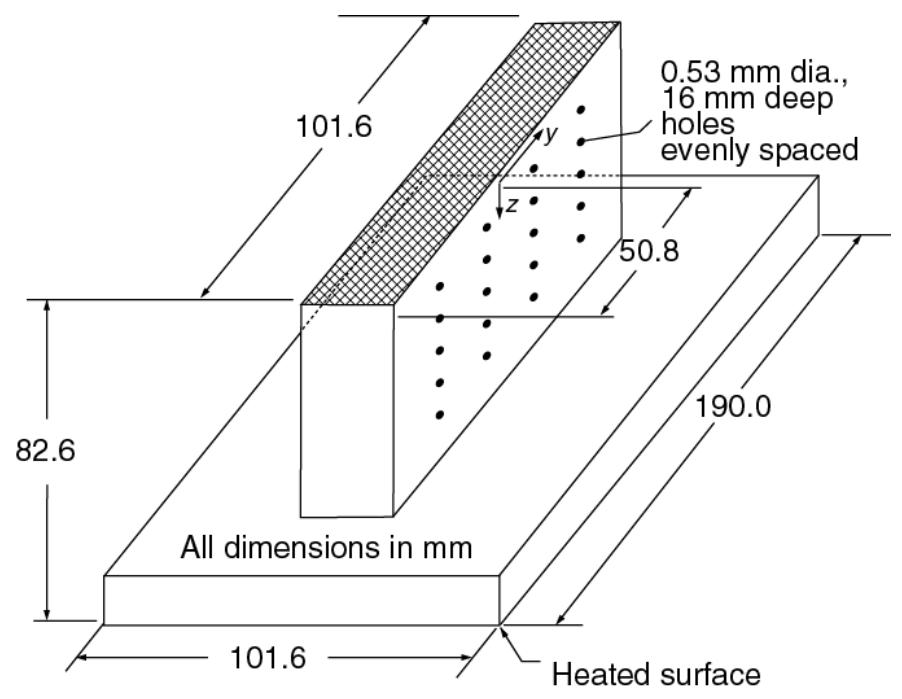

Fig. 2 OFHC copper flat test plate with cross-hatched surface and thermocouple coordinate system

\section{MEASUREMENTS AND UNCERTAINTIES}

The standard uncertainty $\left(u_{i}\right)$ is the positive square root of the estimated variance $u_{i}^{2}$. The individual standard uncertainties are combined to obtain the expanded uncertainty $(U)$, which is calculated from the law of propagation of uncertainty with a coverage factor. All measurement uncertainties are reported at the $95 \%$ confidence level except where specified otherwise. For the sake of brevity, only an outline of the basic measurements and uncertainties is given below. Complete detail on the heat transfer measurement techniques and uncertainties can be found in Kedzierski (2000) and Kedzierski (2009b), respectively.
All of the copper-constantan thermocouples and the data acquisition system were calibrated against a glass-rod standard platinum resistance thermometer (SPRT) and a reference voltage to a residual standard deviation of $0.005 \mathrm{~K}$. Considering the fluctuations in the saturation temperature during the test and the standard uncertainties in the calibration, the expanded uncertainty of the average saturation temperature was no greater than $0.04 \mathrm{~K}$. Consequently, it is believed that the expanded uncertainty of the temperature measurements was less than $0.1 \mathrm{~K}$.

Twenty $0.5 \mathrm{~mm}$ diameter thermocouples were force fitted into the wells of the side of the test plate shown in Fig. 2. The heat flux and the wall temperature were obtained by regressing the measured temperature distribution of the block to the governing two-dimensional conduction equation (Laplace equation). In other words, rather than using the boundary conditions to solve for the interior temperatures, the interior temperatures were used to solve for the boundary conditions following a backward stepwise procedure given in Kedzierski $(1995)^{3}$. The origin of the coordinate system was centered on the surface with respect to the y-direction at the heat transfer surface. Centering the origin in the y-direction reduced the uncertainty of the wall heat flux and temperature calculations by reducing the number of fitted constants involved in these calculations.

Fourier's law and the fitted constants from the Laplace equation were used to calculate the average heat flux $\left(q^{\prime \prime}\right)$ normal to and evaluated at the heat transfer surface based on its projected area. The average wall temperature $\left(T_{\mathrm{w}}\right)$ was calculated by integrating the local wall temperature $(T)$. The wall superheat was calculated from $T_{\mathrm{w}}$ and the measured temperature of the saturated liquid $\left(T_{\mathrm{s}}\right)$. Considering this, the relative expanded uncertainty in the heat flux $\left(U_{q^{\prime \prime}}\right)$ was greatest at the lowest heat fluxes, approaching $10 \%$ of the measurement near $20 \mathrm{~kW} / \mathrm{m}^{2}$. In general, the $U_{q^{\prime \prime}}$ remained approximately between $3 \%$ and $6 \%$ for heat fluxes greater than $50 \mathrm{~kW} / \mathrm{m}^{2}$. The average random error in the wall superheat $\left(U_{\mathrm{Tw}}\right)$ remained between $0.06 \mathrm{~K}$ and $0.14 \mathrm{~K}$. Plots of $U_{q^{\prime \prime}}$ and $U_{\mathrm{Tw}}$ versus heat flux can be found in Kedzierski (2009b).

\section{EXPERIMENTAL RESULTS}

\section{Heat Transfer}

The heat flux was varied approximately between $7 \mathrm{~kW} / \mathrm{m}^{2}$ and $130 \mathrm{~kW} / \mathrm{m}^{2}$ to simulate a range of possible operating conditions for R134a chillers. All pool-boiling tests were taken at 277.6 K saturated conditions. The data were recorded consecutively starting at the largest heat flux and descending in intervals of approximately $4 \mathrm{~kW} / \mathrm{m}^{2}$. The descending heat flux procedure minimized the possibility of any hysteresis effects on the data, which would have made the data sensitive to the initial operating conditions. Kedzierski (2009b) presents the

\footnotetext{
${ }^{3}$ For the record, Table 1 provides functional forms of the Laplace equation that were used in this study in the same way as was done in Kedzierski (1995) and in similar studies by this author.
} 
measured heat flux and wall superheat for all the data of this study.

The mixtures were prepared by charging the test chamber (see Fig. 1) with pure R134a to a known mass. Next, a measured mass of nanolubricant or lubricant was injected with a syringe through a port in the test chamber. The refrigerant/lubricant solution was mixed by flushing pure refrigerant through the same port where the lubricant was injected. All compositions were determined from the masses of the charged components and are given on a mass fraction basis. The maximum uncertainty of the composition measurement is approximately $0.02 \%$, e.g., the range of a $2.0 \%$ composition is between $1.98 \%$ and $2.02 \%$. Nominal or target mass compositions are used in the discussion. For example, the "actual" mass composition of the RL68H in the R134a/ RL68H (99.5/0.5) mixture was $0.51 \% \pm 0.02 \%$. Likewise, the RL68H mass fractions for R134a/ RL68H (99/1) and the R134a/ RL68H (98/2) mixtures were $0.98 \% \pm 0.02 \%$ and $2.00 \%$ $\pm 0.02 \%$, respectively. Using the same uncertainties, the nanolubricant mass fractions as tested with R134a were $0.49 \%, 1.02 \%$, and $1.97 \%$.

Figure 3 is a plot of the measured heat flux $(q ")$ versus the measured wall superheat $\left(T_{\mathrm{w}}-T_{\mathrm{s}}\right)$ for the baseline measurements of the refrigerant/pure-lubricant mixtures at a saturation temperature of $277.6 \mathrm{~K}$. The boiling curve for pure R134a, taken from Kedzierski and Gong (2009), is shown as the leftmost solid line. The open circles, squares, and stars represent the measured heat flux $\left(q^{\prime \prime}\right)$ versus the measured wall superheat $\left(T_{\mathrm{w}}-T_{\mathrm{s}}\right)$ at a saturation temperature of $277.6 \mathrm{~K}$ for the R134a/RL68H (99.5/0.5), R134a/RL68H (99/1), and R134a/RL68H (98/2) mixtures, respectively. Five days of boiling the R134a/RL68H (99.5/0.5) mixture produced 140 measurements over a period of approximately one week. The

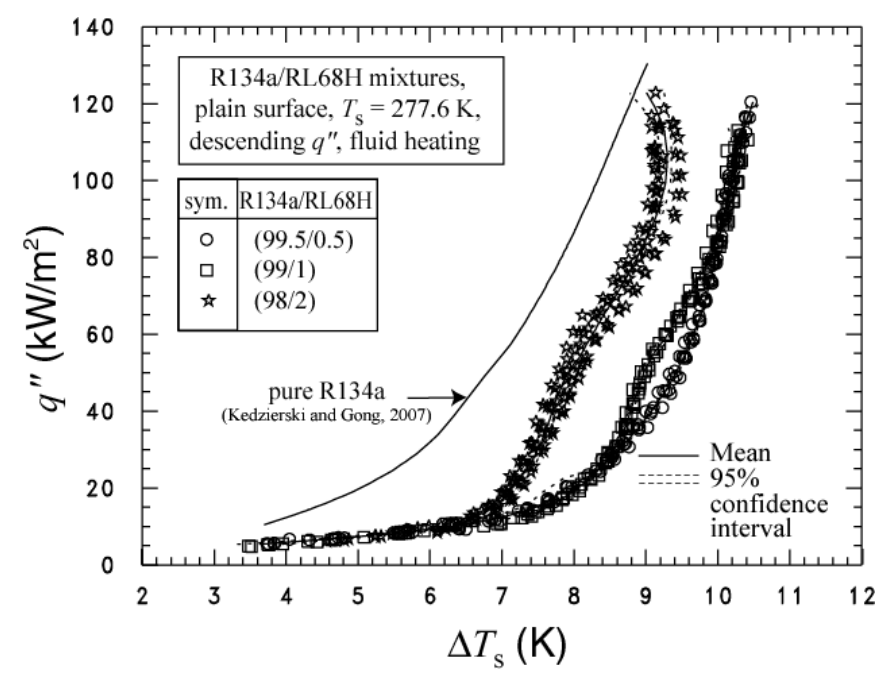

Fig. 3 R134a/RL68H mixtures boiling curves

solid lines shown in Fig. 3 are cubic best-fit regressions or estimated means of the data. Four of the 140 R134a/RL68H (99.5/0.5) measurements were removed before fitting because they were identified as "outliers" based on having both high influence and high leverage (Belsley et al., 1980). Each of the data sets presented here exhibited a similar number of outliers and were regressed in the same manner. Kedzierski (2009b) gives the constants for the cubic regression of the superheat versus the heat flux for all of the fluids tested here. The residual standard deviation of the regressions - representing the proximity of the data to the mean - are given in Kedzierski (2009b). The dashed lines to either side of the mean represent the lower and upper $95 \%$ simultaneous (multiple-use) confidence intervals for the mean. From the confidence intervals, the expanded uncertainty of the estimated mean wall superheat was, on average, $0.11 \mathrm{~K}$ and $0.05 \mathrm{~K}$ for superheats less than and greater than $8 \mathrm{~K}$, respectively. Kedzierski (2009b) provides the average magnitude of $95 \%$ multi-use confidence interval for the fitted wall superheat for all of the test data.

A general overview of the effect that the variation in the pure lubricant mass fraction has on R134a/lubricant pool boiling can be obtained from Fig. 3. Comparison of the three mean boiling curves shows that the superheats are within approximately 1.4 $\mathrm{K}$ of each other for the entire tested heat flux range. For the most part, the superheat for the refrigerant/lubricant mixtures is $1 \mathrm{~K}$ to $3 \mathrm{~K}$ greater than that for pure R134a indicating a heat transfer degradation with respect to pure R134a. Kedzierski (2001b) has shown that, in general, degradations associated with increased lubricant mass fractions occur when the concentration induced bubble size reduction, and its accompanying loss of vapor generation per bubble, is not compensated by an increase in site density. Typically, heat transfer degradations have been observed to increase with respect to increasing lubricant mass fraction. The present measurements are inconsistent with this trend in that the heat transfer performance of the (99.5/0.5) mixture and that of the (99/1) mixture are, for the most part, the same. To add to the inconsistency, the (98/2) mixture exhibits a heat transfer enhancement compared to the (99.5/0.5) and the (99/1) mixtures for heat fluxes greater than $10 \mathrm{~kW} / \mathrm{m}^{2}$. Although the measurements are inconsistent with typical refrigerant/lubricant data, they are fairly repeatable within this study and with respect to the previous study by Kedzierski and Gong (2009). The boiling curves for the present (99.5/0.5) and the (99/1) mixtures agree with those given by Kedzierski and Gong (2009) for the same mixtures on the same surface. However, the superheat for the present (98/2) mixture is approximately $1.5 \mathrm{~K}$ less than what was measured in the previous study for the same conditions. The reason for why only two of the three data sets are repeatable between this study and the Kedzierski and Gong (2009) study is not known. Kedzierski (2001b) has shown that refrigerant/lubricant pool boiling with larger lubricant concentrations typically exhibit greater between-run variability. Also, Kedzierski (2006) has shown that, on occasion, a boiling surface can perform as if it has two different 
sets of active cavities on a given day with one being more favorable for boiling than the other. Considering the above, and without a better explanation, the discrepancy between the two (98/2) data sets over roughly a year between measurements can be explained as a potential characteristic of refrigerant/lubricant boiling with greater lubricant concentrations.

Figures 4 through 6 presents the boiling heat transfer measurements for the three refrigerant/nanolubricant mixtures of R134a and RL68H2C. Figures 4 and 6 show that the R134a/RL68H2C (99.5/0.5) mixture and the R134a/RL68H2C (98/2) mixture both exhibit a significant between-run variation manifested in a stratification of the daily boiling curves. In general, the performance for the (99.5/0.5) and the (98/2) refrigerant/nanolubricant mixtures was stable and repeatable for the first few days; following the stable period, the performance degraded for successive test days. However, the R134a/RL68H2C (99/1) does not exhibit this behavior, i.e., the (99/1) daily boiling curves are random with respect to one another and the data scatter is typical of refrigerant/lubricant boiling. A more detailed discussion of these measurements is given in the following.

Figure 4 shows the measured heat flux $(q ")$ versus the measured wall superheat $\left(T_{\mathrm{w}}-T_{\mathrm{s}}\right)$ for the R134a/RL68H2C (99.5/0.5) mixture at a saturation temperature of $277.6 \mathrm{~K}$. Thirteen boiling curves (one for each test day) were measured over the span of approximately a month. The measurements are separated into three groups as represented by the three different symbols used in the plot. The close circles represent measurement made over the first three days where the surface was initially exposed to diamond nanoparticles. As represented by the plus symbol, successive measurements resulted in an average gain of approximately $0.2 \mathrm{~K}$ in superheat relative to the previous day's measurements, i.e., an increasing degradation in heat transfer performance as the surface experienced more boiling hours. The deterioration of the heat transfer performance with increased usage was believed to be caused by the accumulation of diamond nanoparticles on the surface. To test this hypothesis, the boiling surface was cleaned with acetone and a commercial copper cleaner in an attempt to remove the nanoparticles from the surface. The open circles centered with plus signs represent the boiling measurements that were made over the next three days after the surface was cleaned. The measurements that were made after cleaning agree well with those taken after the first three days of test for heat fluxes greater than roughly $80 \mathrm{~kW} / \mathrm{m}^{2}$. For measurements between approximately $10 \mathrm{~kW} / \mathrm{m}^{2}$ and $80 \mathrm{~kW} / \mathrm{m}^{2}$, the superheat is roughly $0.2 \mathrm{~K}$ less for the cleaned surface than for the first three days of test. All in all, cleaning has restored the heat transfer performance of the boiling surface. The solid line along with confidence intervals documents the fit of the six best test runs, i.e., three boiling curves after the initial run plus three boiling curves after cleaning the surface. The average expanded uncertainty of the estimated mean wall superheat for the fitted refrigerant/nanolubricant mixture was $0.23 \mathrm{~K}$ and
$0.13 \mathrm{~K}$ for superheat greater than and less than $6 \mathrm{~K}$,

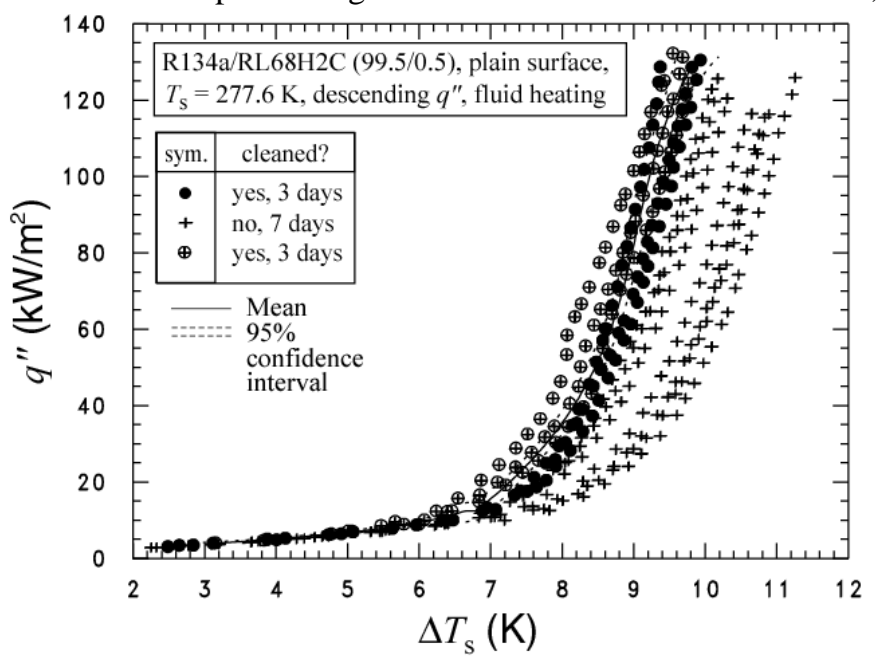

Fig. 4 R134a/RL68H2C (99.5/0.5) mixture boiling curves

respectively.

Figure 5 shows the measured heat flux $(q ")$ versus the measured wall superheat $\left(T_{\mathrm{w}}-T_{\mathrm{s}}\right)$ for the R134a/RL68H2C (99/1) mixture at a saturation temperature of $277.6 \mathrm{~K}$. Eight boiling curves were measured over the span of approximately two weeks. Unlike the R134a/RL68H2C (99.5/0.5) measurements, the measurements for the (99/1) mixture exhibited a modest random variation in performance rather than a continuous degradation with respect to test day. For most heat fluxes, the R134a/RL68H2C (99/1) superheat measurements, represented by the closed squares, agree well with the six best measurement days for the R134a/RL68H2C $(99.5 / 0.5)$ mixture. In fact, the mean boiling curve for the (99.5/0.5) mixture differs from the mean for the (99/1) mixture by no more than approximately $0.2 \mathrm{~K}$. The average expanded uncertainty of the estimated mean wall superheat for the fitted

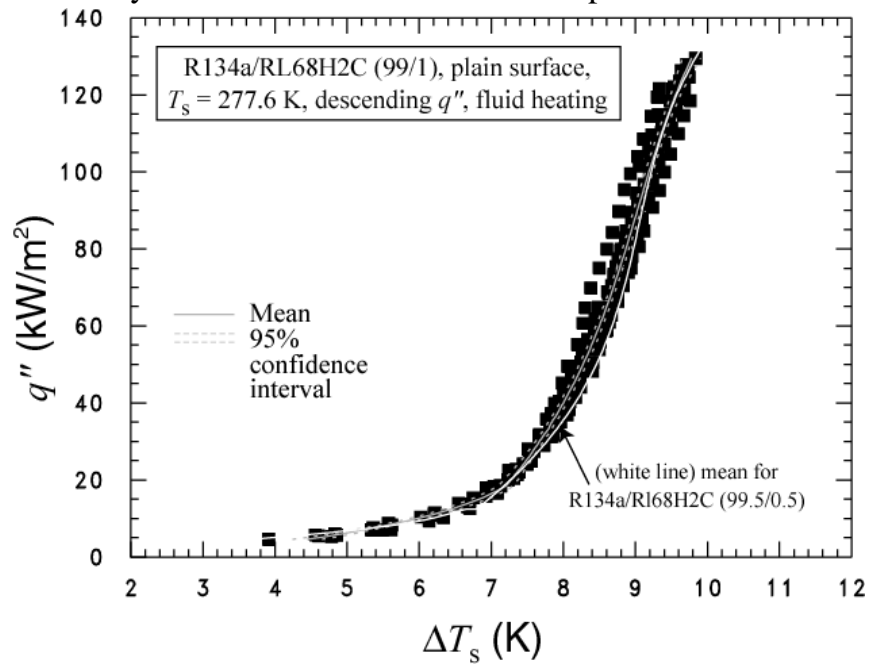

Fig. 5 R134a/RL68H2C (99/1) mixture boiling curves 
refrigerant/nanolubricant mixture was $0.12 \mathrm{~K}$ and $0.06 \mathrm{~K}$ for superheat greater than and less than $8 \mathrm{~K}$, respectively.

Figure 6 shows the measured heat flux $(q ")$ versus the measured wall superheat $\left(T_{\mathrm{w}}-T_{\mathrm{s}}\right)$ for the R134a/RL68H2C (98/2) mixture at a saturation temperature of $277.6 \mathrm{~K}$. Ten boiling curves were measured over the span of approximately three weeks. The measurements for the (98/2) refrigerant/nanolubricant mixture exhibit a behavior similar to that of the measurements for the (99.5/0.5) refrigerant/nanolubricant mixture. The first four days of measurement for the (98/2) mixture, as represented by the closed stars, are fairly repeatable and random between runs. Consequently, the first four days were regressed together and are shown as the solid and dashed lines representing the mean and $95 \%$ confidence interval, respectively. The average expanded uncertainty of the estimated mean wall superheat for the fitted refrigerant/nanolubricant mixture was $0.17 \mathrm{~K}$ and $0.10 \mathrm{~K}$ for superheat greater than and less than $7 \mathrm{~K}$, respectively. The plus symbols show measurements taken after the first four days at a degraded performance. The open circles centered with plus signs represent the boiling measurements that were made after cleaning the surface. Cleaning restored the performance of the surface for higher heat fluxes for one run, but had essentially no effect on the performance for another run.

A more precise comparison of the R134a/RL68H and the R134a/RL68H2C heat transfer performances relative to R134a and R134a/RL68H, respectively, is given in Figs. 7 and 8. Figure 7 plots the ratio of the R134a/RL68H mixture heat flux to the pure R134a heat flux $\left(q{ }_{\mathrm{PL}} / q{ }_{\mathrm{p}}\right)$ versus the pure R134a heat flux $\left(q^{\prime \prime}\right)$ at the same wall superheat. Figure 7 illustrates the influence of lubricant mass composition on the $\mathrm{R} 134 \mathrm{a} / \mathrm{RL} 68 \mathrm{H}$ boiling curve with solid lines representing the mean heat flux ratios for each mixture and shaded regions showing the $95 \%$ confidence level for the mean. Overall, lubricant for all compositions has caused a heat transfer degradation relative to the heat transfer of pure R134a for all measured $q_{p}{ }_{p}$. The refrigerant/lubricant pool boiling heat flux is shown to be between roughly $20 \%$ and $60 \%$ of that of the

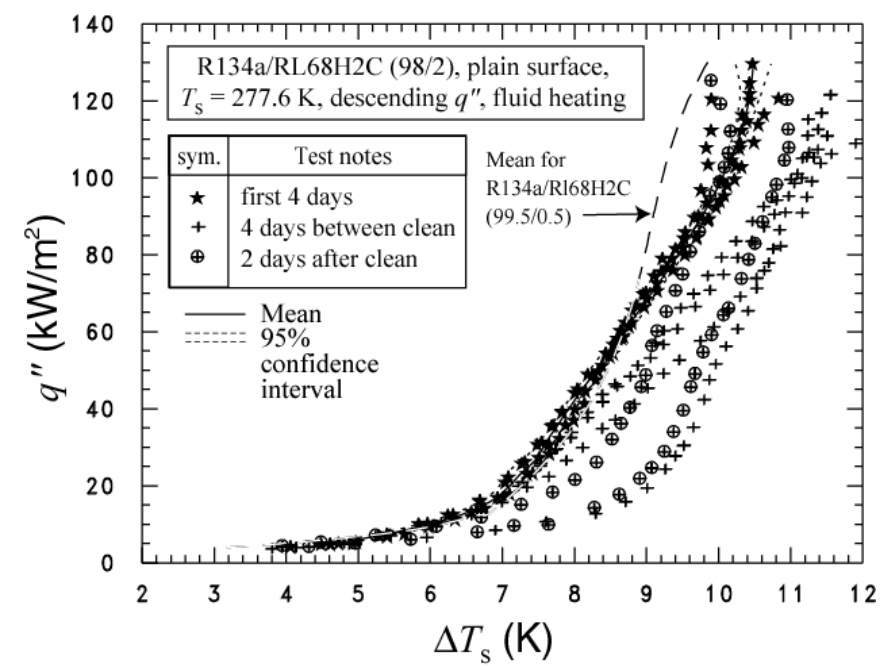

Fig. 6 R134a/RL68H2C (98/2) mixture boiling curves pure refrigerant. The minimum performance for each

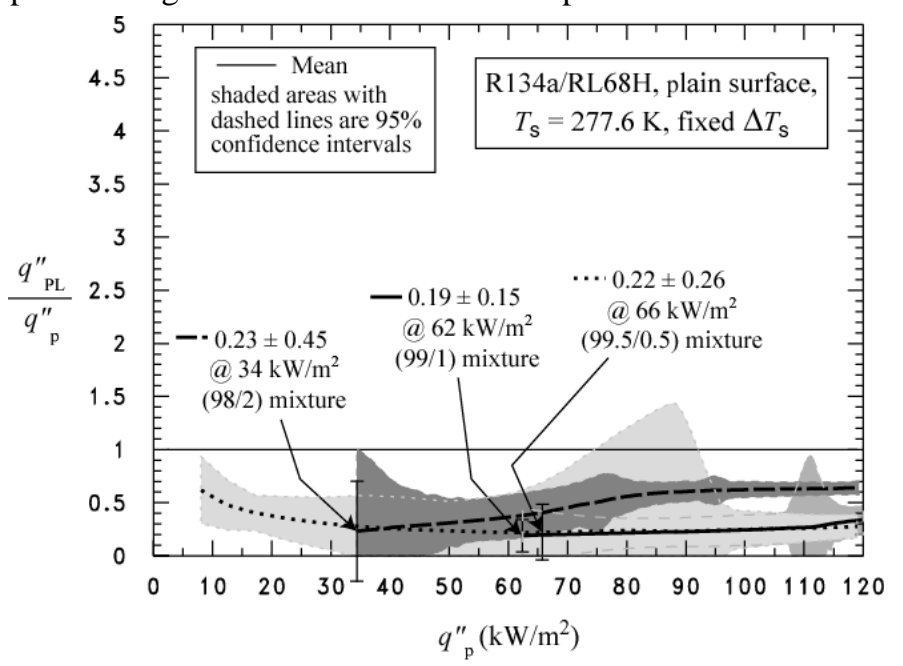

Fig. 7 Boiling heat flux of R134a/RL68H mixture relative to that of pure $\mathrm{R} 134 \mathrm{a}$

refrigerant/lubricant mixture is approximately $20 \%$ of the performance of pure R134a. For example, at roughly $64 \mathrm{~kW} / \mathrm{m}^{2}$, the minimum heat flux ratio for the R134a/RL68H (99.5/0.5), and the R134a/RL68H (99/1) mixture was $0.22 \pm 0.26$, and $0.19 \pm 0.15$, respectively. The minimum heat flux ratio for the R134a/RL68H (98/2) mixture was $0.23 \pm 0.45$ at approximately $34 \mathrm{~kW} / \mathrm{m}^{2}$. Overall, the average heat flux ratio for the R134a/RL68H (99.5/0.5), the R134a/RL68H (99/1), and the R134a/RL68H (98/2) mixture for the heat flux range shown in Fig. 7 for each fluid was 0.27, 0.24, and 0.48, respectively.

Figure 8 details the effect that the diamond nanoparticles had on the R134a/RL68H boiling curves for the repeatable, best performance runs as described in the discussion of Figs 4 and 6. The figure plots the ratio of the R134a/RL68H2C heat flux to the R134a/RL68H heat flux $\left(q{ }^{\prime}{ }_{\mathrm{C}} / q{ }_{\mathrm{PL}}\right)$ versus the

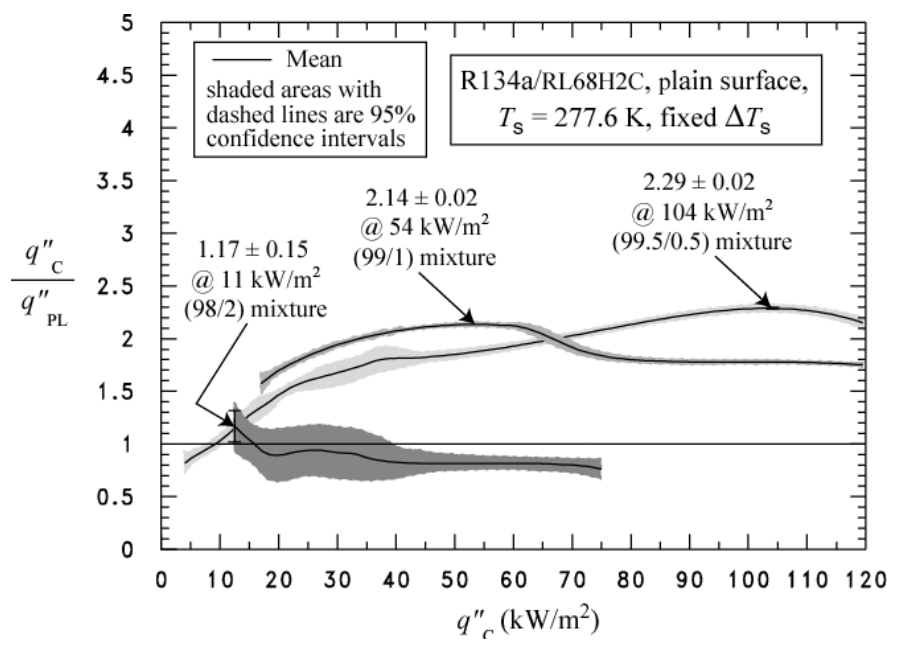

Fig. 8 Boiling heat flux of R134a/RL68H2C mixtures relative to that of $R 134 a / R L 68 H$ without nanoparticles for best performance data 
R134a/RL68H2C mixture heat flux $\left(q^{\prime \prime}\right.$ ) at the same wall superheat. The three different compositions are represented by three different lines where each R134a/nanolubricant mixture is compared to the R134a/pure-lubricant mixture at the same mass fraction. A heat transfer enhancement exists where the heat flux ratio is greater than one and the $95 \%$ simultaneous confidence intervals (depicted by the shaded regions) do not include the value one. Figure 8 shows that the R134a/RL68H2C (99.5/0.5) and (99/1) mixtures exhibit a significant boiling heat transfer enhancement over that of the R134a/RL68H (99.5/0.5) and the R134a/RL68H (99/1) mixtures (without nanoparticles), respectively. The heat flux ratio varies between roughly 0.8 and 2.29 for the R134a/RL68H2C (99.5/0.5) mixture for heat fluxes between $4 \mathrm{~kW} / \mathrm{m}^{2}$ and $120 \mathrm{~kW} / \mathrm{m}^{2}$. The R134a/RL68H2C (99/1) mixture shows a maximum heat flux ratio of approximately 2.14 at approximately $54 \mathrm{~kW} / \mathrm{m}^{2}$. The R134a/RL68H2C (98/2) mixture shows a maximum heat flux ratio of approximately 1.17 and a region between $11 \mathrm{~kW} / \mathrm{m}^{2}$ and $40 \mathrm{~kW} / \mathrm{m}^{2}$ where no difference can be established between the two fluids because the confidence intervals include the value of one. Overall, the average heat flux ratio for the R134a/RL68H2C (99.5/0.5) mixture and the R134a/RL68H2C (99/1) mixture from approximately $15 \mathrm{~kW} / \mathrm{m}^{2}$ to $120 \mathrm{~kW} / \mathrm{m}^{2}$ was 1.98 , and 1.91 , respectively. The average heat flux ratio for the R134a/RL68H2C (98/2) mixture from approximately 11 $\mathrm{kW} / \mathrm{m}^{2}$ to $75 \mathrm{~kW} / \mathrm{m}^{2}$ was 0.81 .

Given that the boiling curves showed a surface aging effect for the (99.5/0.5) and the (98.2) mixtures with nanoparticles, Fig. 9 provides the heat flux ratios, as done in Fig. 8, but for the two worst performance runs shown in Figs 4 and 6. In this way, Figs. 8 and 9 can be used to bracket the observed performance for tests conducted with nanoparticles in this

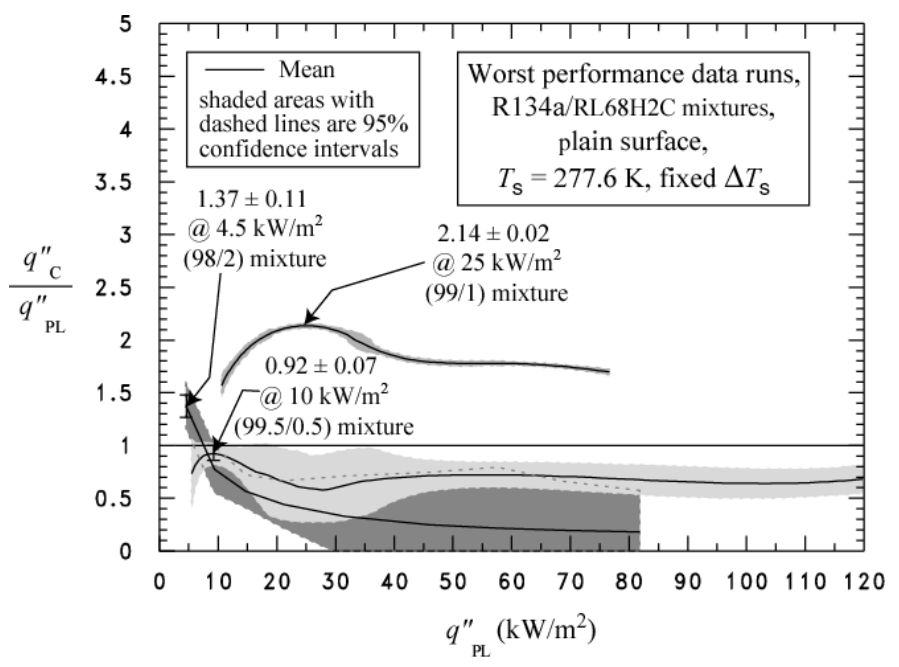

Fig. 9 Boiling heat flux of R134a/RL68H2C mixtures relative to that of $\mathrm{R} 134 \mathrm{a} / \mathrm{RL68H}$ without nanoparticles for worst performance data runs study. ${ }^{4}$ The heat flux ratio for the R134a/RL68H2C (99/1) mixture is the same as was presented in Fig. 8 with the exception that it is plotted against the refrigerant/pure-lubricant heat flux rather than the refrigerant/nanolubricant heat flux. The maximum heat flux ratio for worst performing R134a/RL68H2C mixtures was $0.92 \pm 0.07$ and $1.37 \pm 0.11$ for the (99.5/0.5) and the (98/2) mixture, respectively. Averaged for heat fluxes between approximately $5 \mathrm{~kW} / \mathrm{m}^{2}$ and $80 \mathrm{~kW} / \mathrm{m}^{2}$, the heat flux ratio for the worst performing R134a/RL68H2C runs for the (98/2) and the (99.5/0.5) mixtures was 0.36 and 0.76 , respectively.

\section{Particle Size}

The size of the diamond nanoparticles in the nanolubricant was measured with a Dynamic Light Scattering (DLS) technique using a $633 \mathrm{~nm}$ wavelength laser and a sieving technique using a syringe filter and an optical microscope. The two methods were required because the diamond nanolubricant was very polydispersed and contained particles that were larger than what could be measured with the DLS technique.

The DLS technique was used to measure particles sizes less than $1000 \mathrm{~nm}$. Approximately $0.01 \mathrm{~g}$ of the nanolubricant was mixed with approximately $3 \mathrm{~g}$ of toluene and pushed through a $1 \mu \mathrm{m}$ syringe filter into the sample cuvette that was analyzed with the DLS system. An index of refraction of 2.42 for diamond was used in the Brownian motion based calculation that was done internally by the DLS instrument for the particle size. The uncertainty of the packaged DLS instrumentation was confirmed with a NIST traceable $60 \mathrm{~nm}$ nanofluid standard. The measured diameter of the standard with the DLS system was within $5 \mathrm{~nm}$ of range of uncertainty of the standard. For the diamond nanolubricant, for particles smaller than $1 \mu \mathrm{m}$, the $10 \mathrm{~nm}$ diameter ${ }^{5}$ diamond nanoparticles were agglomerated primarily into two clumps of $30 \mathrm{~nm}$ and $400 \mathrm{~nm}$ with a bandwidth of approximately $\pm 60 \%$ for each of approximately equal intensities. Consequently, for particles smaller than $1 \mu \mathrm{m}$, the nanolubricant had nearly an equal number of nearly discrete diamond nanoparticles (single or clumps of 2 to 5 particles), and agglomerated clumps of approximately 40 particles.

The $1 \mu \mathrm{m}$ syringe filter material used above was examined with an optical microscope. Figure 10 shows the diamond particles that can be seen in the filter are between roughly $10 \mu \mathrm{m}$ and $50 \mu \mathrm{m}$. However, particle agglomeration as large as $250 \mu \mathrm{m}$ was observed when the mixture was left to settle over several weeks. Jillavenkatesa et al. (2001) show that it would require thousands of size measurements to obtain an uncertainty in the size distribution for a $95 \%$ confidence level. Consequently, only an approximate range for the particle size is given here.

\footnotetext{
${ }^{4}$ Because of the consistent trend of the stratified data, it is likely that a larger degradation would have been exhibited by the worst performing mixtures had more data runs been taken.

${ }^{5}$ The $10 \mathrm{~nm}$ particle diameter was specified by the manufacturer of the nanopowder that was used to make the nanolubricant.
} 


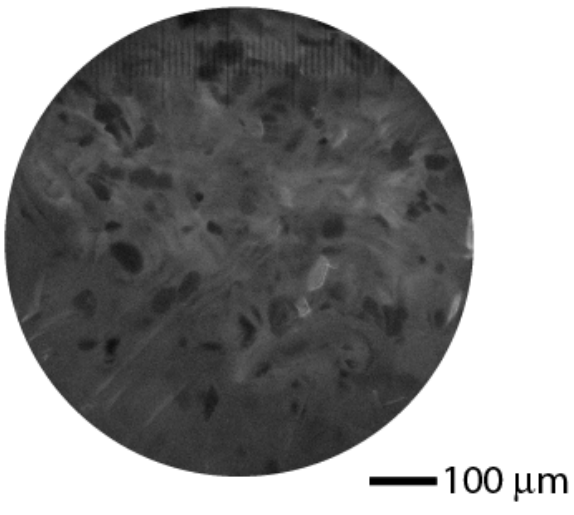

Fig. 10 Agglomerated diamond nanoparticles in syringe filter material

Combining the results of the two measurement methods shows that the nanolubricant is not a good dispersion. The particles are dispersed from single $10 \mathrm{~nm}$ diameter particles to agglomerations of particles as large as $50 \mu \mathrm{m}$. The large clumps of particles are expected to settle and further agglomerate as demonstrated by the $250 \mu \mathrm{m}$ agglomerations observed in the aged sample. In other words, the diamond nanolubricant dispersion made in this study was not stable and experienced settling over time.

\section{DISCUSSION}

As far as refrigerant boiling is concerned, there are two direct consequences that an agglomerated nanolubricantdispersion has on heat transfer. The first is that the polydispersed nanolubricant has a very large viscosity: approximately $450 \mu \mathrm{m}^{2} / \mathrm{s}$ at $313.15 \mathrm{~K}$, which is nearly a $550 \%$ increase over the viscosity of the pure lubricant (see Kedzierski, 2009b). Kedzierski (2001b) showed that increases in lubricant viscosity resulted in improvements in boiling heat transfer. From this, it is shown below that the viscosity increase of the diamond nanolubricant is largely responsible for the boiling heat transfer enhancement associated with this particular refrigerant/nanolubricant mixture. The second consequence of a poor dispersion is that it can cause a heat transfer instability if the particles are large enough to settle over time despite the vigorous mixing as caused by the boiling.

A previous study (Kedzierski, 2009) suggested that nanoparticle interaction with bubbles was the main boiling enhancement mechanism of boiling refrigerant/CuOnanolubricant mixtures. In order for the interaction to occur, the nanoparticles must remain suspended in the lubricant excess layer that resides on the boiling surface. Nanoparticles that become lodged in surface cavities are likely to degrade heat transfer performance via loss of bubble nucleation sites. Particles that are not governed by Brownian motion, i.e., particles that are too large, are not likely to provide favorable and stable interaction with bubbles. Over time, the larger particles are likely to agglomerate and settle onto the boiling surface. The worsening performance over time exhibited by the (99.5/0.5)-nanolubricant mixture and the (98/2)nanolubricant mixture is speculated to be a direct result of particle settling over time.

The above argument suggests that nanoparticle interaction with bubbles is not likely to be responsible for the heat transfer enhancements that were observed for the refrigerant/diamondnanolubricant mixtures. Considering that diamondnanoparticle interaction is not the governing heat transfer enhancement mechanism, then is it possible for increased nanolubricant viscosity to be the cause? In an attempt to demonstrate that it is, Fig. 11 compares the enhancement ratio for the R134a/nanolubricant mixtures to those predicted by the refrigerant/lubricant mixture, pool-boiling model given in Kedzierski (2003). The dashed lines of Fig. 11 are predictions from the refrigerant/lubricant pool boiling model while using the properties of the refrigerant and the measured fluid properties of the nanolubricant (see Kedzierski, 2009b) at the $2.6 \%$ diamond volume fraction. Overall, the model predicts the relative stratification of the boiling measurements for the three mixtures with the smaller nanolubricant mass fractions having the best improvement. In addition, fairly good absolute agreement was achieved between measurements and predictions for each nanolubricant mass fraction. For example, the heat flux ratios for the $(99.5 / 0.5)$ mixture and the (98/2) mixture were overpredicted, on average, by roughly $5 \%$ and $26 \%$, respectively. Similarly, the (99/1) mixture is underpredicted by about $25 \%$ on average. Given that the model cannot account for nanoparticle interaction with bubbles, the agreement suggests that the boiling heat transfer enhancement with the nanolubricant of this study was due

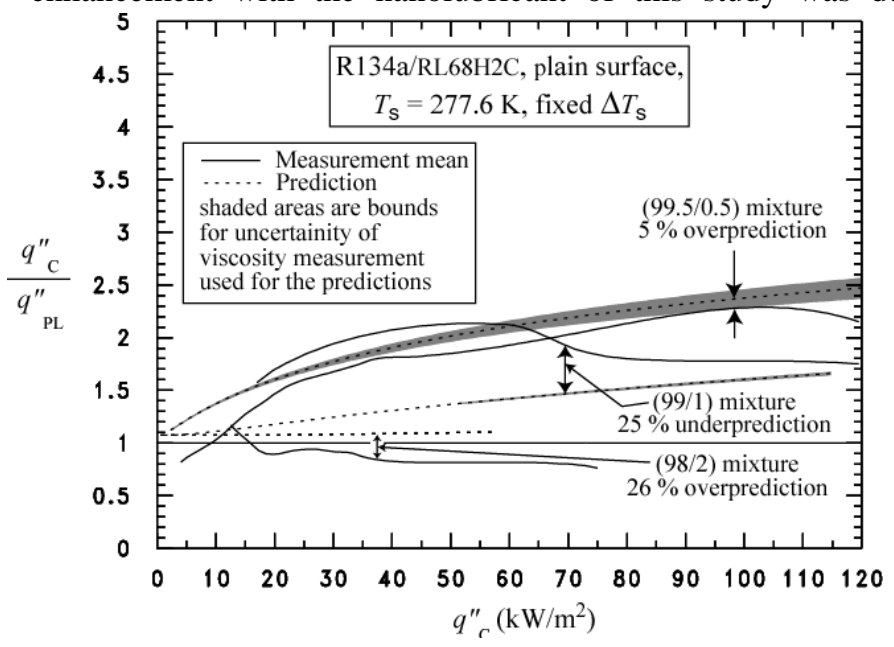

Fig. 11 Predicted heat flux ratio for $\mathrm{RL68H} 2 \mathrm{C}$ (99.5/0.5) mixture using Kedzierski (2003) model compared to measurement means 
mainly to favorable changes in fluid properties, i.e., increases in liquid viscosity and thermal conductivity ${ }^{6}$ with respect to the pure lubricant.

At this point it is appropriate to address the stable performance of the R134a/RL68H2C (99/1) mixture and how it relates to the above discussion. It was noted by Jillavenkatesa et al. (2001) that the particle distribution in a nanofluid has the potential for significant variation. Considering this and the wide range of particle size of the diamond nanolubricant of this study, it may have been possible that the nanolubricant that was used to make the R134a/RL68H2C (99/1) mixture had less particle agglomeration than what was used to make the other two mixtures even though it was taken from the same batch. If this were the case, there would be less particle settling, which would lead to greater measurement repeatability between runs. In addition, the boiling heat transfer performance was on average $25 \%$ greater than what was predicted by the model based on refrigerant/lubricant properties. Potentially, that $25 \%$ difference, if it is not due to prediction errors, could be due to particle interaction as caused by a better nanofluid suspension.

Future research is required to investigate the influence of the particle material, its shape, size, distribution, and concentration on refrigerant boiling performance. In particular, the importance of having a good dispersion in promoting nanoparticle-bubble interaction should be verified with further refrigerant boiling studies with well-dispersed diamond nanolubricants. Sarkas (2009) suggests that this may be more easily said than done because the surface chemistry of diamond may be well suited for encouraging particle agglomeration. As a result, the surface chemistry of diamond nanoparticles must be altered (eg., hydrogen-atom terminated bonds, Sarkas (2009)) before it can become a viable material for chiller nanolubricants. Further investigation in this area and with other nanoparticle materials may lead to a theory that can be used to develop nanolubricants that improve boiling heat transfer for the benefit of the refrigeration and air-conditioning industry.

\section{CONCLUSIONS}

The effect of diamond nanoparticles on the boiling performance of R134a/polyolester mixtures on a roughened, horizontal flat surface was investigated. A nanolubricant containing diamond nanoparticles at $2.6 \%$ volume fraction with a polyolester lubricant was mixed with R134a at three different mass fractions. Because the nanolubricant was polydispersed with relatively large agglomerated particles, the boiling heat transfer performance was shown to degrade with time for two out of three of the test mixtures and was likely due to particles filling nucleation sites. As a result, measurement comparisons were made for both the worst and the best-case heat transfer performances. For the best case, the $0.5 \%$ nanolubricant mass fraction, the diamond nanoparticles caused

\footnotetext{
${ }^{6}$ As shown in Kedzierski (2009b), the nanoparticles resulted in an approximate $7 \%$ increase in the thermal conductivity of that of the pure lubricant.
}

a heat transfer enhancement relative to the heat transfer of pure R134a/polyolester (99.5/0.5) on average of $98 \%$. A similar enhancement was observed for the R134a/nanolubricant (99/1) mixture, which had a heat flux that was on average $91 \%$ larger than that of the R134a/polyolester (99/1) mixture. Further increase in the nanolubricant mass fraction to $2 \%$ resulted in a boiling heat transfer degradation of approximately $19 \%$ on average for the R134a/nanolubricant (98/2) mixture. For the worst case, the R134a/nanolubricant (98/2) mixture and the R134a/nanolubricant (99.5/0.5) mixture exhibited a $64 \%$ and a $24 \%$ degradation, respectively. The R134a/nanolubricant (99/1) mixture performance did not degrade over time, and as a result exhibited a consistent $91 \%$ enhancement.

From the results of the present study, it is speculated that if a good dispersion of nanoparticles in the lubricant is not obtained, then the agglomerated nanoparticles will not provide interaction with bubbles, which is favorable for heat transfer. The clumps of nanoparticles can provide heat transfer enhancement via purely fluid property effects, i.e., increases in liquid viscosity and thermal conductivity. These property improvements persist only as long as the particles remain suspended in the lubricant excess layer. Reductions in boiling heat transfer performance increase as the agglomerated nanoparticles settle out of the excess layer and into the cavities of the boiling surface. For this reason, unless the surface chemistry of the diamond nanoparticles can be altered, diamond-nanolubricants do not appear to be a viable material for chiller applications.

The present study demonstrates the necessity of having the particles well dispersed in the nanolubricant so that the particles remain suspended to provide a favorable performance and induce interaction with bubbles for further boiling heat transfer enhancement.

\section{ACKNOWLEDGEMENTS}

This work was funded by NIST. Thanks go to the following NIST personnel for their constructive criticism of the first draft of the manuscript: Mr. B. Dougherty, and Dr. P. Domanski. Thanks go to Dr. D. Olson of NIST for his constructive criticism of the second draft of the manuscript. Furthermore, the author extends appreciation to Mr. W. Guthrie and Mr. A. Heckert of the NIST Statistical Engineering Division for their consultations on the uncertainty analysis. Boiling heat transfer measurements were taken by Mr. David Wilmering of KT Consulting at the NIST laboratory. The RL68H (EMKARATE RL 68H) was donated by Dr. S. Randles of Uniqema. 


\section{REFERENCES}

Bahrami, M., Yovanovich, M. M., and Culham, J. R., 2006, "Assessment of Relevant Physical Phenomena Controlling Thermal Performance of Nanofluids," Proceedings of IMECE2006, Chicago, IMECE2006-13417.

Baumeister, T., Avallone, E. A., and Baumeister, T., 1978, "Marks' Standard Handbook for Mechanical Engineers," $8^{\text {th }}$ ed., McGraw-Hill, NY, pp. 4-63.

Belsley, D. A., Kuh, E., and Welsch, R. E., 1980, Regression Diagnostics: Identifying Influential Data and Sources of Collinearity, New York: Wiley.

Bi, S., Shi, L., and Zhang, L., 2007, Application of Nanoparticles in Domestic Refrigerators, Applied Thermal Engineering, Vol. 28, pp. 1834-1843.

Bobbo, S., Fedele, L., Fabrizio, M., Barison, S., Battiston, S., Pagura C., 2009, "Influence of Nanoparticles Dispersion in POE Oils on Lubricity and R134a Solubility," Proceedings of $3^{\text {rd }}$ IIR Conference on Thermophysical Properties and Transport Processes of Refrigerants, Boulder CO., paper IIR176.

Energy Information Administration (EIA), 2008, “2003

Commercial Buildings Energy Consumption Survey:

Consumption and Expenditures Tables,"

http://www.eia.doe.gov/emeu/cbecs/cbecs2003/detailed_tables

_2003/2003set15/2003pdf/c13a.pdf (January 2009).

Environmental Protection Agency (EPA), 2008, "EPA

Green Building Strategy,” EPA-100-F-08-073.

Jillavenkatesa, A., Dapkunas, S. J., Lum, L. H., 2001, “Particle Size Characterization,” NIST Special Publication 960-1, U.S. Department of Commerce, Washington, D.C.

Kedzierski, M. A., 2009a, "Effect of CuO Nanoparticle Concentration on R134a/Lubricant Pool-Boiling Heat Transfer," ASME J. Heat Transfer, Vol. 131, No. 4, 043205.

Kedzierski, M. A., 2009b, "Effect of Diamond Nanolubricant on R134a Pool Boiling Heat Transfer with Extensive Measurement and Analysis Details,” NIST Technical Note XXXX, U.S. Department of Commerce, Washington, D.C.

Kedzierski, M. A., and Gong, M., 2009, "Effect of CuO Nanolubricant on R134a Pool Boiling Heat Transfer with Extensive Measurement and Analysis Details,” Int. J. Refrigeration, Vol. 25, pp. 1110-1122.

Kedzierski, M. A., 2006, "A Comparison of R245fa Pool Boiling Measurements to R123, and R245fa/Isopentane on a Passively Enhanced, Horizontal Surface,” I. J. Trans. Phenomena, Vol. 8, No. 4, pp. 331-344.

Kedzierski, M. A., 2003, "A Semi-Theoretical Model for Predicting R123/Lubricant Mixture Pool Boiling Heat Transfer," Int. J. Refrigeration, Vol. 26, pp. 337-348.

Kedzierski, M. A., 2002, "Use of Fluorescence to Measure the Lubricant Excess Surface Density During Pool Boiling,” Int. J. Refrigeration, Vol. 25, pp. 1110-1122.

Kedzierski, M. A., 2001a, "Use of Fluorescence to Measure the Lubricant Excess Surface Density During Pool Boiling,” NISTIR 6727, U.S. Department of Commerce, Washington, D.C.
Kedzierski, M. A., 2001b, "The Effect of Lubricant Concentration, Miscibility and Viscosity on R134a Pool Boiling” Int. J. Refrigeration, Vol. 24, No. 4., pp. 348-366.

Kedzierski, M. A., 2000, "Enhancement of R123 Pool Boiling by the Addition of Hydrocarbons,” Int. J. Refrigeration, Vol. 23, pp. 89-100.

Kedzierski, M. A., 1995, "Calorimetric and Visual Measurements of R123 Pool Boiling on Four Enhanced Surfaces," NISTIR 5732, U.S. Department of Commerce, Washington.

Lee, S., Choi, S., Li, S, and Eastman, J., A., 1999, "Measuring Thermal Conductivity of Fluids Containing Oxide Nanoparticles,” ASME J. Heat Transfer, Vol. 121, pp. 280-289.

Liu, M. S., Lin, M.C.C., Liaw, J.S., Hu, R. Wang, C.C., 2009, "Performance Augmentation of a Water Chiller System Using Nanofluids," Proceedings of ASHRAE Winter Conference, Chicago, CH-09-058.

Marquis, F. D. S., and Chibante, L. P. F., 2005, "Improving the Heat Transfer of Nanofluids and Nanolubricants with Carbon Nanotubes,” J. Minerals, Metals and Materials Society, Vol. 57, No. 12, pp. 32-43.

Office of Science and Technology Policy (OSTP), 2008,

"Federal Research and Development Agenda for Net-Zero Energy, High-Performance Green Buildings,” National Science and Technology Council Committee on Technology, http://www.ostp.gov/galleries/

NSTC\%20Reports/FederalRDAgendaforNetZeroEnergyHighP erformanceGreenBuildings.pdf (March 2009).

Roder, H. M., Perkins, R. A., Laesecke, A., and Nieto de Castro, C. A., 2000, “Absolute Steady-State Thermal Conductivity Measurements by Use of a Transient Hot-Wire System,” J. Res. Natl. Inst. of Stand. and Technol., Vol. 105, No. 2, pp. 221-253.

Sarkas, H., 2009, Private Communications, Nanophase Technologies Corporation, Romeoville, IL.

Vadasz, P., 2006, "Heat Conduction in Nanofluid Suspensions,” ASME J. Heat Transfer, Vol. 128, pp. 465-477.

Wasp, F. J., 1977, "Solid-Liquid Flow Slurry Pipeline Transportation, Trans., Tech. Pub., Berlin.

Wolter, S. D., Borca-Tasciuc, D. A., Chen, G., Govindaraju, N., Collazo, R., and Okuzumi, F., 2003, "Thermal Conductivity of Epitazially Textured Diamond Films,” Diamond Related Materials, Vol. 12, pp. 61-64. 